\title{
Learning in the cloud: a new challenge for a global teaching system in optics and photonics
}

Razia Sultana, Andreas Christ, Markus Feisst, Dan Curticapean

Razia Sultana, Andreas Christ, Markus Feisst, Dan Curticapean, "Learning in the cloud: a new challenge for a global teaching system in optics and photonics," Proc. SPIE 9289, 12th Education and Training in Optics and Photonics Conference, 92891R (17 July 2014); doi: 10.1117/12.2070558

SPIE Event: 12th Education and Training in Optics and Photonics Conference, 2013, Porto, Portugal 


\title{
Learning in the cloud: A new challenge for a global teaching system in optics and photonics
}

\author{
Razia Sultana $^{1,}$ Andreas Christ ${ }^{1}$, Markus Feisst ${ }^{2}$, Dan Curticapean ${ }^{1}$, \\ ${ }^{1}$ University of Applied Sciences Offenburg, Germany, dan.curticapean@hs-offenburg.de \\ ${ }^{2}$ Meyle+Müller GmbH+Co.KG, mfeisst@meyle-mueller.de
}

Keywords: Education, Mobile-learning, education in optics and photonics, content adaption

\begin{abstract}
Nowadays, it is assumed of many applications, companies and parts of the society to be always available online. However, according to [Times, Oct, 31 2011], 73\% of the world population do not use the internet and thus aren't "online" at all. The most common reasons for not being "online" are expensive personal computer equipment and high costs for data connections, especially in developing countries that comprise most of the world's population (e.g. parts of Africa, Asia, Central and South America). However it seems that these countries are leap-frogging the "PC and landline" age and moving directly to the "mobile" age. Decreasing prices for smart phones with internet connectivity and PC-like operating systems make it more affordable for these parts of the world population to join the "always-online" community. Storing learning content in a way accessible to everyone, including mobile and smart phones, seems therefore to be beneficial. This way, learning content can be accessed by personal computers as well as by mobile and smart phones and thus be accessible for a big range of devices and users. A new trend in the Internet technologies is to go to "the cloud". This paper discusses the changes, challenges and risks of storing learning content in the "cloud". The experiences were gathered during the evaluation of the necessary changes in order to make our solutions and systems "cloud-ready".
\end{abstract}

\section{Introduction}

In general cloud services is used either as storage or as a service provider such as computing. At the end it is to reduce IT operational costs by outsourcing hardware and software maintenance and support to the cloud provider. In this paper we are proposing a combined approach of using cloud services for academic purposes specially for mobile learning. The goal of this research work is to consider mobility of learner, heterogeneity of end users 'devices along with diversity of learning content for Optics and photonics.

According to CISCO IBSG, (April 2011) by 2020 approximately 50 billion connected devices will be used by approximately 7.6 billion of world population, which means 6 to 8 connected device per person. It seems to be very advantageous if those devices could be used to provide and receive learning content for optics and photonics from the cloud for leaning purposes.

\section{Background}

The underlying concept of cloud computing dates back to the 1950s, when large scale mainframe became available in academia and corporations, accessible via thin clients / terminal computers, often referred to as "dumb terminals", because they were used for communications but had no internal computational capacities. To make more efficient

12th Education and Training in Optics and Photonics Conference, edited by

Manuel F. P. C. Martins Costa, Mourad Zghal, Proc. of SPIE Vol. 9289, 92891R

(c) 2014 SPIE, OSA, IEEE, ICO · doi: 10.1117/12.2070558

Proc. of SPIE Vol. 9289 92891R-1 
use of costly mainframes, a practice evolved that allowed multiple users to share both the physical access to the computer from multiple terminals as well as to share the CPU time. This eliminated periods of inactivity on the mainframe and allowed for a greater return on the investment. The practice of sharing CPU time on a mainframe became known in the industry as time-sharing. [1]

In the 1990s, telecommunications companies, who previously offered primarily dedicated point-to-point data circuits, began offering virtual private network (VPN) services with comparable quality of service, but at a lower cost. By switching traffic as they saw fit to balance server use, they could use overall network bandwidth more effectively. They began to use the cloud symbol to denote the demarcation point between what the provider was responsible for and what users were responsible for. Cloud computing extends this boundary to cover servers as well as the network infrastructure. [2]

As computers became more prevalent, scientists and technologists explored ways to make large scale computing power available to more users through time sharing, experimenting with algorithms to provide the optimal use of the infrastructure, platform and applications with prioritized access to the CPU and efficiency for the end users. [3]

\section{Challenges}

To support mobile learning for optics and photonics with the help of cloud, first it has to be clear what we consider as mobile learning. Kinshuk et al. (2003) [4] propose an appropriate definition of Mobile Learning: "m-learning is defined as the ability of using handheld devices to access learning resources"; All humans have the right to access learning materials and information to improve their quality of life regardless of where they live, their status, and their culture. Mobile learning, through the use of mobile technology, will allow citizens of the world to access learning materials and information from anywhere and at anytime. This idea will literally come true only after a worldwide successful implementation of presentation of device independent learning content. The identified barriers that have to be taken care of to support mobile learning are:

1) Various kinds of devices used by different users or in different times or parallel by the same user. In a stable place like at home or at office it is more convenient to use a PC. While on the move it is very obvious that a user would like to access same content with the same outlook and feel by using his mobile device. 2) The overall number of users of specialized content or interactive applications is too low to adapt the application or content to all possible devices manually. 3) Content and User Interfaces could include different kinds of data format like text, image, audio, video, 3D Virtual Reality data and upcoming other formats [5]. The system should be able to deal with all the existing and upcoming formats of data without requiring any huge enhancement. 4) In ideal case the content should be always available to the user independent of the internet access possibility. Moreover in case of multiple devices, used by the same user, should be synchronized to make updated content available in all the used devices. The goal of this research work is to find a way to solve the above stated problems.

\section{Potentials}

For academic purposes professors or learning content providers are responsible to prepare and upload contents. While preparing them the author should have this freedom to upload any kind of data and not to think about end

user's device. The students, who are the end users, should be able to access the learning material independent of his device, time and place. Based on the connectivity all of the used devices have to be synchronized to provide up dated learning material any time anywhere. Figure 1 is showing the simplified version of the process. 

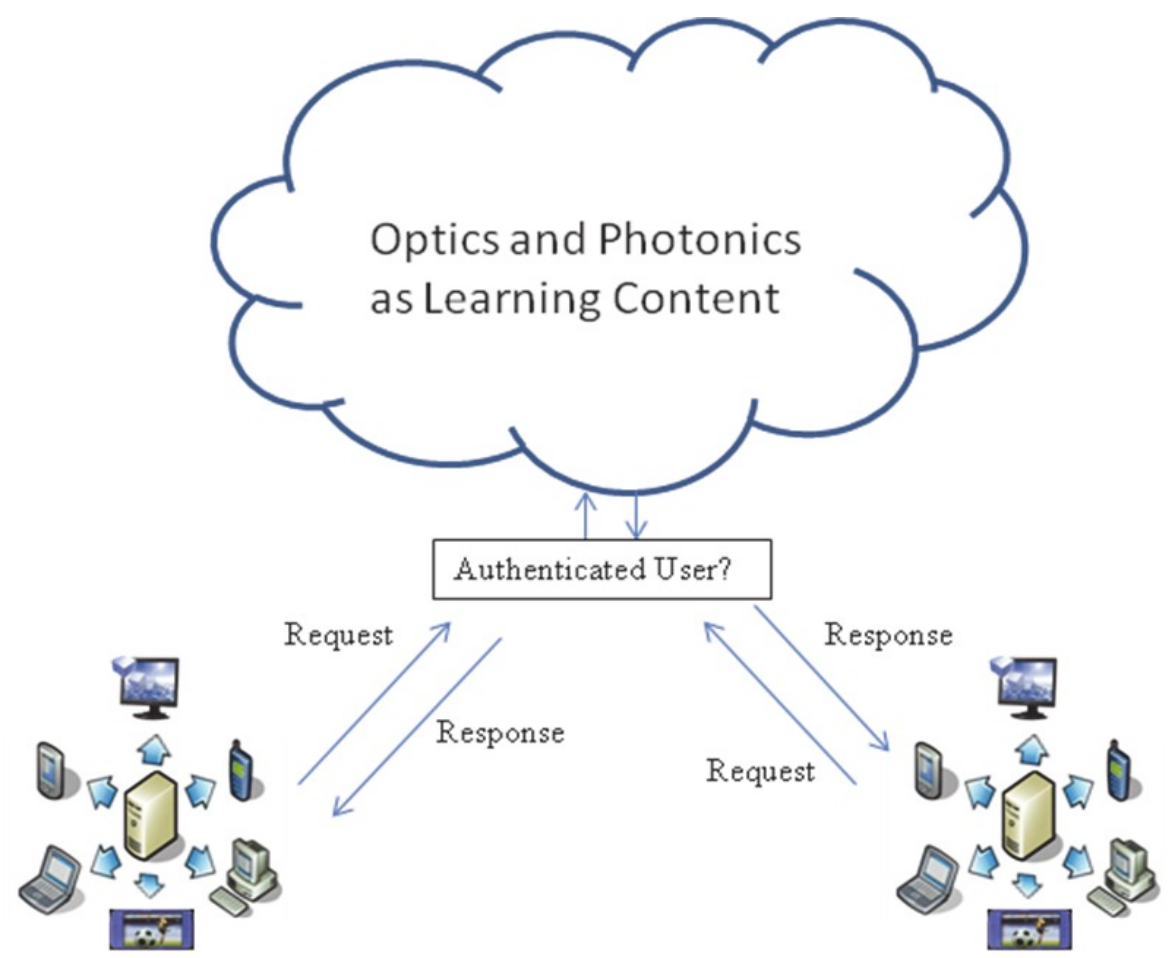

User A having multiple device, being synchronized with each other

User B having multiple device, being synchronized with each other

Figure 1. A simplified version of System Architecture.

Now let us consider an example of optics and photonics as learning content, where the author has explained about Aspherical lense, its advantages over classical spherical lense, numerical equation to derive and simulate aspherical lense. For better understanding of the students an animated simulation is also uploaded in the cloud server. The animated simulation is is not only helping students to understand the concept better but also making a result easily accessible which is very costly to produce due to expensive simulation tool and higher processing power to use the tool.

\section{Content example - Aspherical lense}

Years ago lenses were build with spherical surfaces, this kind of lenses have very high spherical aberration. Due to this high aberration the quality of image decreases dramatically. Today there is a possibility to use ray-tracing to compute an optimal surface and the technology to manufacture lenses that have this computed surface is available as well. This computed surfaces are called aspherical surface, depicted in figure 2 


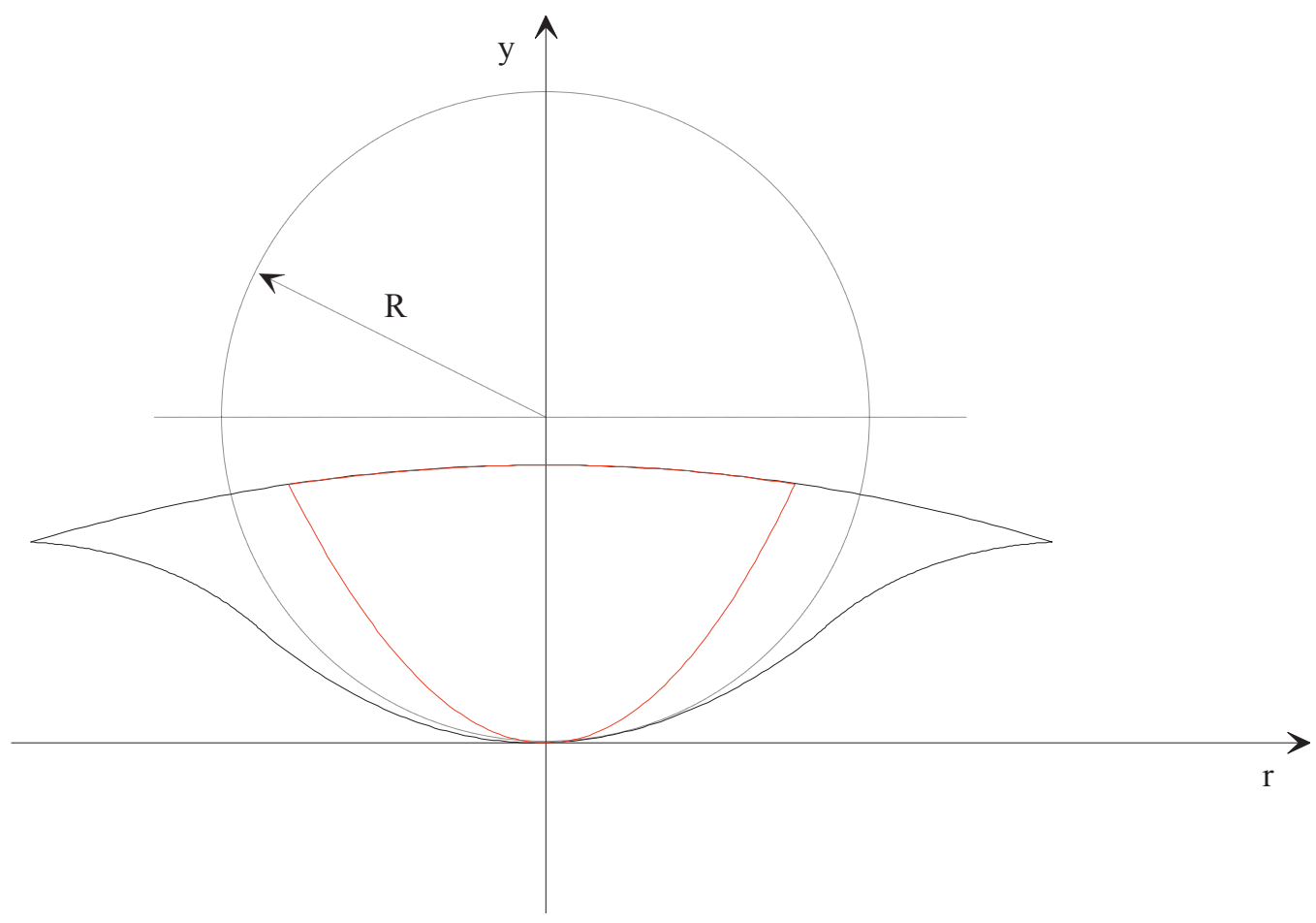

Fig. 2: Aspheric lens - geometric overview

Lens consisting of aspherical surface/s are called Aspherical lens. This is essential ingredient in reducing the sizes and the weights of optical systems. It is said that one aspherical lens can be as good as 3 to 4 spherical lenses. One rather common reason for employing an aspherical lens is to eliminate spherical aberration.

The equation below describes aspherical surface introduced by [6][7]

$$
y(r)=\frac{r^{2}}{R\left(1+\sqrt{1-(1+\kappa)\left(\frac{r}{R}\right)^{2}}\right)}
$$

In the above mentioned equation the type of the surface is given by $\mathrm{k}$. If we consider the parameter $\mathrm{k}<-1$ the surface is hyperbola (figure 3 ) for $\mathrm{k}=-1$ it is parabola and for $-1<\mathrm{k}<0$ the surface is ellipse (figure 4 ). The classical spherical surfaces is formed by $\mathrm{k}=0$. For all the positives value of $\mathrm{k}$ will form ellipse. 


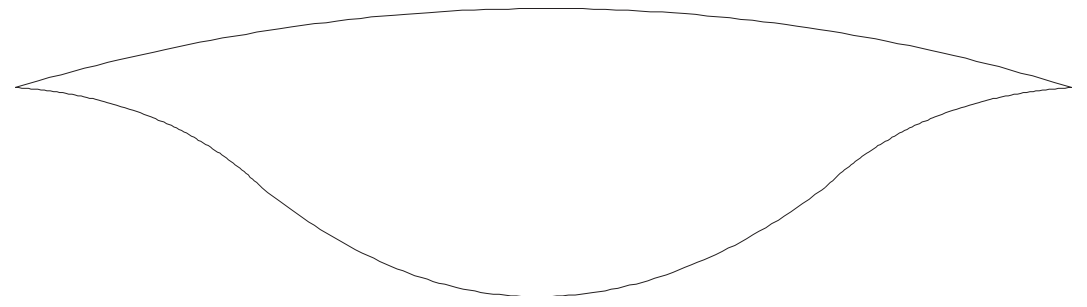

Fig. 3: Aspheric lens with hyperbola surface

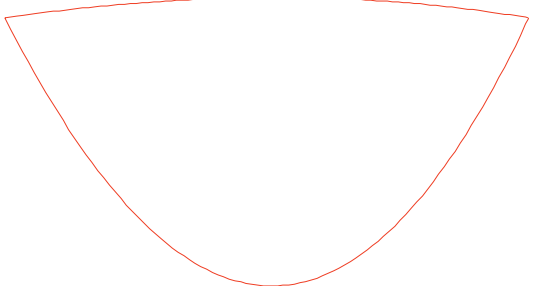

Fig. 4: Aspheric lens with elliptical surface

The animated simulation below shows the influence of $\mathrm{k}$ on the surface form (figure 5).

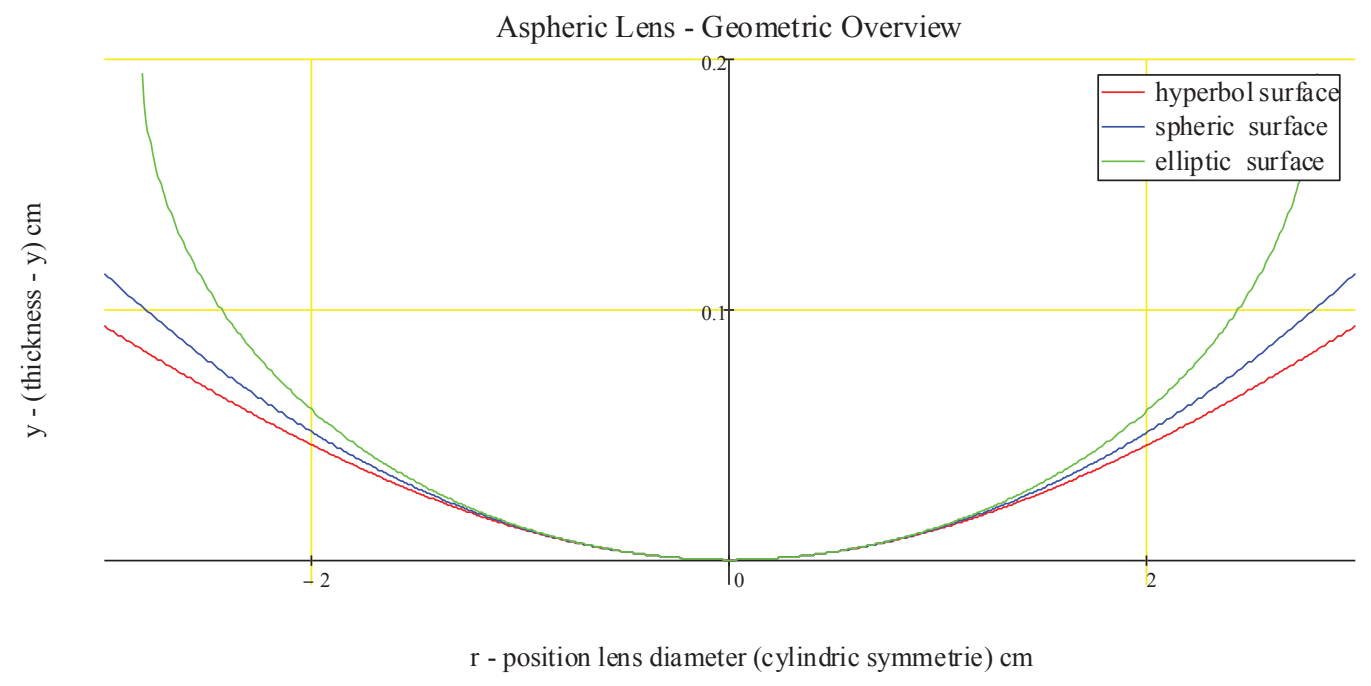

Fig. 5: Animated simulation of aspherical surfaces

Proc. of SPIE Vol. 9289 92891R-5 


\section{Risk}

As with other changes in the landscape of computing, certain legal issues arise with cloud computing, including trademark infringement, security concerns and sharing of proprietary data resources.

One important but not often mentioned problem with cloud computing is the problem of who is in "possession" of the data. If a cloud company is the possessor of the data, the possessor has certain legal rights. If the cloud company is the "custodian" of the data, then a different set of rights would apply. The next problem in the legalities of cloud computing is the problem of legal ownership of the data. Many Terms of Service agreements are silent on the question of ownership. [8]

These legal issues are not confined to the time period in which the cloud based application is actively being used. There must also be consideration for what happens when the provider-customer relationship ends. In most cases, this event will be addressed before an application is deployed to the cloud. However, in the case of provider insolvencies or bankruptcy the state of the data may become blurred. [9]

\section{Open questions}

From the user -end nobody knows where exactly the provided data is saved. Who else has access to this data? For Our provided Optics and Photonics learning content is open for registered student of the course only. Should it be open for rest of the world? If yes how can we respect legal terms and conditions of different nations? Should the user also have the right to modify the provided learning content to make the learning process more interactive and collaborative? If yes how can we assure the reliability of the content? The synchronization is being done among different devices used by the same user. Is it also advantageous to be synchronized with other user's device? In this regard the question of reliability arises again. Moreover who is responsible to which part of the data?

Above stated questions are only examples of some of the open issues which have to be considered to make the best use of learning in the cloud.

\section{Conclusion}

It is a great challenge but the effort to find answers is worth due to the immense benefits of reaching the goal- allow citizens of the world to access learning materials and information from anywhere and at anytime. Our approach towards the solution is research on different software architecture by implementing state of the art technologies such as XML, object oriented database-Db4o, hibernate etc in a small scale in the prototype [10][11][12].

Nowadays, cloud services are offering a high flexibility at a low cost. Thus, with a few clicks it is possible to add required resources such as more processing power or more storage. There is no need to invest in hardware or in staff; the investment can be used directly for research and education. Additionally, there is no need to apply the different security rules of different organization/institutions, which makes it easy to share results, data and services with everyone if desired. That makes different institutions able to cooperate in creating learning material for their students and combine the knowledge.

Nevertheless, storing data in the cloud has drawbacks as well. The question of who owns the intellectual property of material and data and responsibility of copyright infringement. The question "where the data is physically stored" sometimes cannot be answered because this might be dependent on the cloud service provider. This fact might cause problems in case of the influence of different national laws. However, the possibility to use cloud services offers a big potential for education purpose and national as well as international cooperation. 


\section{Reference}

[1] Strachey, Christopher (June 1959). "Time Sharing in Large Fast Computers".Proceedings of the International Conference on Information processing, UNESCO. paper B.2.19: 336-341.

[2] "July, 1993 meeting report from the IP over ATM working group of the IETF". CH: Switch. Retrieved 22 Aug 2010.

[3] Corbató, Fernando J. "An Experimental Time-Sharing System". SJCC Proceedings. MIT. Retrieved 3 July 2012.

[4] Kinshuk, Suhonen, J., Sutinen, E., Goh, T., (2003), Mobile Technologies in Support of Distance Learning, Asian Journal of Distance Education, 1(1):60-68

[5] (Meawad F ; Stubbs, G.(2008), A framework for enabling on-demand personalised mobile learning, International Journal of Mobile Learning and Organisation, 2(2):133-148

[6] Gyeong-Il Kweon,_Cheol-Ho Kim: Aspherical Lens Design by Using a Numerical Analysis; Journal of the Korean Physical Society, Vol. 51, No. 1, July 2007, pp. 93 103

[7] Gross, H., Blechinger, F., Achtner, B., (2008), Handbook of Optical Systems, WILEY-VCH Verlag GmbH \& Co. KGaA

[8] Maltais, Michelle (26 April 2012). "Who owns your stuff in the cloud?". Los Angeles Times. Retrieved 14 Dec 2012.

[9] Chambers, Don (July 2010). "Windows Azure: Using Windows Azure's Service Bus to Solve Data Security Issues]". Rebus Technologies. Retrieved 14 Dec 2012.

[10] Christ, A., Feisst, M., (2010). SW_Architecture for device independent mobile learning. In Caballe, S; Xhafa, F.; Daradoumis, T. \& Juan, A. A. (Eds.), Architectures for Distributed and Complex M-Learning Systems: Applying Intelligent Technologies, (pp. 72-93) USA, IGI Global

[11] Christ, A., Feisst, M. \& Sultana, R., (2012) Collaborative language learning game as a device independent application. In Isaias, P., et al. (Eds.), Towards Learning and Instruction in Web 3.0: Advances in Cognitive and Educational Psychology, (pp. 73-88), USA, Springer

[12] Christ, A., Meyrueis, P., \& Sultana, R. (in press). Architecture of a device independent collaborative language learning game. In Z.L. Berge \& L.Y. Muilenburg (Eds.), Handbook of Mobile Learning. New York: Routledge 